
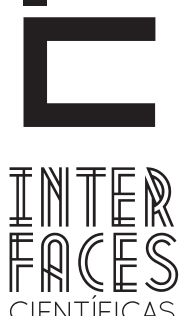

HUMANASE SOCIAIS

ISSN IMPRESSO 2316-3348

E-ISSN 2316-3801

DOI - 10.17564/2316-3801.2018v6n3p93-100

\title{
MENSURAÇÃO DOS NÍVEIS DE ANSIEDADE TRAÇO E ESTADO EM ESTUDANTES DO CURSO DE ENFERMAGEM
}

\author{
MEASUREMENT OF TRAIT AND STATE ANXIETY LEVELS IN NURSING COURSE STUDENTS \\ MEDICIÓN DE LOS NIVELES DE ANSIEDAD RASGO Y ESTADO DE ESTUDIANTES EN CURSO DE ENFERMERÍA
}

Fernanda Mirelly Freitas Menezes

Lívia Melo Barros

Hugo Márcio Rodrigues de Almeida ${ }^{5}$

\author{
Victor Levi Rocha Rodrigues ${ }^{2}$ \\ André Faro ${ }^{4}$ \\ Paulo Autran Leite Lima ${ }^{6}$
}

\section{RESUMO}

Ao estudar a ansiedade, duas vertentes podem ser utilizadas, na qual uma delas trata-se do estado emocional transitório, caracterizado por sentimentos subjetivos de tensão que podem variar em intensidade ao longo do tempo que é definida como ansiedade-estado. $E$ a outra é referentea uma disposição pessoal, relativamente estável, a responder com ansiedade a situações estressantes e uma tendência a perceber um maior número de situações como ameaçadoras, denominada como ansiedade-traço. A pesquisa teve como objetivo mensurar os níveis de ansiedade traço e ansiedade, estado em estudantes do curso de Enfermagem frente à disciplina de Anatomia Humana I. Tratou-se de um estudo quantitativo de corte transversal no qual foi aplicado o Inventário de ansiedade traço e estado (IDATE) uma semana antes da primeira avaliação da disciplina, numa Universidade particular da cidade de Aracaju-SE. Foi pos- sível observar que a ansiedade traço foi estatisticamente significativa $(p=0,02)$, e a ansiedade estado não $(p=0,14)$. Quando comparados à variável eventos ocorridos, os níveis de ansiedade traço e estado foram significativos $(p=0,001)$, no entanto, não houve alteração estatística quando relacionou-se a ansiedade traço e estado a outras diferentes variáveis. De acordo com a metodologia utilizada, pode-se concluir que a disciplina de anatomia não gerou ansiedade significativa nos discentes e que a ansiedade traço foi significativa independente das variáveis analisadas.

\section{PALAVRAS-CHAVE}

Medicina do Comportamento. Estudantes de Enfermagem. Ansiedade. 


\section{ABSTRACT}

When studying anxiety, two strands can be used, one of which is the transient emotional state, characterized by subjective feelings of tension that may vary in intensity over time that is defined as anxiety-state. The other is about a relatively stable personal disposition to respond with anxiety to stressful situations and a tendency to perceive a greater number of situations as threatening, termed anxiety-trait. The aim of the research was to measure the levels of anxiety trait and anxiety state in students of the Nursing course against the discipline of Human Anatomy I. It was a cross-sectional quantitative study in which the State-Trait Anxiety Inventory (IDATE) a week before the first evaluation of the subject, at a private university in the city of Aracaju (SE). It was possible to observe that the trace anxiety was statistically significant $(p=$ $0.02)$ and the anxiety state was not $(p=0.14)$. When compared to the variable events occurred, levels of trace and state anxiety were significant $(p=0.001)$. However, there was no statistical change when trace and state anxiety was related to other different variables. Thus, according to the methodology used, it can be concluded that the anatomy discipline did not generate significant anxiety in the students and that the trace anxiety was significant independent of the analyzed variables.

\section{KEYWORDS}

Behavioral medicine. Nursing students. Anxiety.

\section{RESUMEN}

Mediante el estudio de la ansiedad, dos vertientes se pueden utilizar, en el que uno es el estado emocional transitorio caracterizado por sentimientos subjetivos de tensión que pueden variar en intensidad con el tiempo que se define como el estado de ansiedad. El otro está relacionado con la disposición personal, relativamente estables con ansiedad la respuesta a situaciones de estrés, y una tendencia a percibir un mayor número de situaciones como una amenaza, que se refiere a la ansiedad rasgo. El estudio tuvo como objetivo medir niveles de rasgos de ansiedad y estado de ansiedad en los estudiantes del curso de enfermería a través de la disciplina de la anatomía humana. Se trata de un estudio transversal cuantitativo en el que se aplicó un cuestionario inventario de ansiedad rasgo y estado (IDATE), una semana antes de la primera evaluación del curso, en una universidad privada en la ciudad de Aracaju (SE). Se

observó que la ansiedad rasgo fue estadísticamente significativa $(p=0,02)$, y no el estado de ansiedad ( $p$ $=0,14)$. Cuando se compara con los acontecimientos se produjeron variables, los niveles de ansiedad rasgo y estado fueron significativas ( $p=0,001$ ), sin embargo, no hubo ningún cambio estadística cuando relacionado con la ansiedad rasgo y estado a otras variables diferentes. De acuerdo con la metodología utilizada, se puede concluir que la disciplina académica de anatomía no generó ansiedad significativa en los estudiantes y que la ansiedad rasgo eran variables independientes significativas analizadas.

\section{PALABRAS CLAVE}

La conducta médica. Estudiantes de enfermería. Ansiedad. 


\section{INTRODUÇ̃̃O}

A ansiedade é uma condição individual e desagradável causada por alterações psico-fisiológicas como resultado de confrontos com situações que confundem, causam medo, irritam ou ameaçam o individuo. 0 organismo reage ao estimulo que é interpretado pelo individuo como ameaça, perturbando a homeostase e causando um desequilíbrio emocional e físico, tendo manifestações como: taquicardia, hipertensão, fadiga, cefaleia, dentre outros (CARMO et al.; LEITE et al., 2016).

A ansiedade pode ser estudada a partir de duas modalidades de funcionamento adaptativo, sendo elas a ansiedade traço e a ansiedade estado. A ansiedade estado está relacionada a reações emocionais desagradáveis, singularizado por sentimentos de apreensão e preocupação, decorrentes de um estado momentâneo e passageiro. Já a ansiedade traço é caracterizada pelas diferenças individuais, com tendência para identificar situações estressantes como ameaçadoras e perigosas, e reagir de maneira peculiar a sua personalidade (CORDEIRO, 2016).

Um dos desafios que provoca dificuldades de estabelecer vínculos afetivos entre os acadêmicos é a ansiedade, e devido a isso, gera problemas de adaptação social. Estudos revelam que graduandos de cursos da área da saúde sofrem de estresse e ansiedade, e no decorrer da universidade esses níveis tendem a aumentar (CARVALHO et al., 2015), além disso, outros estudos indicam que acadêmicos de enfermagem estão mais expostos a eventos estressantes em razão das situações de responsabilidade pela vida e saúde dos indivíduos (HIRSCH et al., 2015).

Ao realizar uma pesquisa bibliográfica sobre os níveis de ansiedade traço e estado no contexto acadêmico, tanto na esfera nacional quanto internacional, utilizando como base as plataformas Scielo, BVS Psicologia e Pepsic, podemos perceber a escassez de pesquisas que abordem o tema proposto. Visto isso, o presente estudo teve como objetivo geral mensurar os níveis de ansiedade-traço e ansiedade-estado em estudantes do curso de Enfermagem, a fim de efetivar e agregar conhecimentos as pesquisas que abordem essa tese no país.

\section{MATERIAIS E MÉTODOS}

Tratou-se de um estudo quantitativo de corte transversal, no qual o Inventário de Ansiedade Traço Estado (IDATE) foi aplicado uma semana antecedente à primeira avaliação em todos os alunos que cursavam a disciplina de anatomia humana I do curso de Enfermagem em uma universidade privada do município de Aracaju-SE.

O Inventário é composto por 20 questões presentes em cada escala, cujo nível de resposta varia entre 1 a 4, em cada escala do tipo Likert. A obtenção do escore é feito a partir da soma dos itens, executando-se o recorde dos itens positivos. A resultante são os escores de ansiedade-traço e ansiedade-estado obtidos em cada escala. Além do IDATE, também foi aplicado um questionário sociodemográfico composto pelas variáveis: gênero, idade e por informações a respeito da vida acadêmica dos indivíduos, constando questões como período atual, se já cursou a disciplina de anatomia anteriormente e se já é formado em curso de nível técnico ou superior.

Inicialmente foram realizadas análises exploratórias, a fim de verificar a adequação dos dados ao modelo normal, bem como ajustes de digitação e perdas amostrais. Em seguida, foram obtidas as análises descritivas, o que inclui o cálculo dos escores das escalas, conforme orientação do próprio protocolo dos instrumentos. Na análise inferencial trabalhou-se com duas variáveis dependentes (VD) (ansiedade-traço e ansiedade-estado), sendo que as variáveis independentes (VI) são as demais características cotejadas no estudo (sociodemográficas e relativas ao contexto escolar). Além disso, foram executados testes de associação e comparação de médias por VD a outros estudos existentes que utilizaram como método o IDATE. Todas as análises foram realizadas com o auxílio do software SPSS (v. 21), utilizando o teste t de Student e nível de significância de $p<0,05$. 
A pesquisa foi aprovada pelo Comitê de Ética em Pesquisa (CEP) da Universidade Tiradentes (UNIT), tendo como Certificado de Apresentação para Apreciação Ética (CAAE) a numeração 39609114.8.0000.5371. Os voluntários foram previamente informados sobre os procedimentos do estudo, incluindo os possíveis riscos e benefícios necessários. Após a leitura e concordância, os alunos assinaram o Termo de Consentimento Livre e Esclarecido de acordo com a Resolução 466/12 e suas complementares do Conselho Nacional de Saúde.

\section{RESULTADOS E DISCUSSÃO}

Participaram da pesquisa 148 alunos do primeiro período do curso de enfermagem que estavam cursando a disciplina de anatomia humana. Sendo que destes, 38 foram do gênero masculino (26\%) e 110 do sexo feminino (74\%). A média da ansiedade estado em relação ao gênero masculino foi de 48,39 $\pm 1,84$ e no gênero feminino foi de $51,33 \pm 0,99$. Já a média da ansiedade traço no gênero masculino foi de 43,81 11,70 e no gênero feminino foi de 47,88 $\pm 0,88$, dados disponíveis na Tabela 1 .

Além da variável gênero, outras variáveis foram analisadas para verificar se houve uma relação com a ansiedade. Relacionando aos eventos ocorridos previamente, como, por exemplo, doenças, término de relacionamento, falecimento de familiares ou amigos, entre outros, 90 (61\%) indivíduos relataram não ter ocorrido nada significativo naquele período, com média de $47 \pm 1,13$ na ansiedade estado e 44,63 $\pm 1,00$ na ansiedade traço e 58 (39\%) indivíduos relataram que algum evento ocorreu previamente, tendo como média na ansiedade estado 54,63 $\pm 1,24$ e na ansiedade traço $50,25 \pm 1,19$.

Quanto aos alunos que já cursaram a disciplina, foi visto que 116 (78\%) alunos estavam cursando pela primeira vez, possuindo média de $51,18 \pm 0,98$ na ansiedade estado e 47,25 $\pm 0,90$ na ansiedade traço, justificando a média de idade encontrada que foi de 21,10 $\pm 4,41$ anos e 32 (22\%) discentes já haviam cursado a disciplina, com média de 48,37 $\pm 1,97$ na ansiedade estado e 45,31 $\pm 1,71$ na ansiedade traço.

Foi visto, tratando-se da variável de alunos formados em algum curso técnico na área da saúde, que 119 (80\%) discentes relataram não possuir nenhuma formação, possuindo média de 50,88 $\pm 0,94$ na ansiedade estado e 47,18 $\pm 0,88$ na ansiedade traço, enquanto que 29 (20\%) informaram possuir formação de nível técnico na área da saúde, assumindo média de 49,34 $\pm 2,28$ na ansiedade estado e 45,41 $\pm 1,90$ na ansiedade traço. Os dados correspondentes as variáveis analisadas podem ser visualizados na Tabela 2.

Tabela 1 - Ansiedade traço e estado correlacionada com os gêneros

\begin{tabular}{|c|c|c|c|c|c|}
\hline & Gênero & $\mathbf{N}$ & $\%$ & Média \pm e.p. & $\mathbf{P}$ \\
\hline Ansiedade estado & $\begin{array}{l}\text { Masculino } \\
\text { Feminino }\end{array}$ & $\begin{array}{l}38 \\
110\end{array}$ & $\begin{array}{l}26 \% \\
74 \%\end{array}$ & $\begin{array}{c}48,39 \pm 1,84 \\
51,33 \pm 0,99\end{array}$ & 0,14 \\
\hline Ansiedade traço & $\begin{array}{l}\text { Masculino } \\
\text { Feminino }\end{array}$ & $\begin{array}{l}38 \\
110\end{array}$ & $\begin{array}{l}26 \% \\
74 \%\end{array}$ & $\begin{array}{l}43,81 \pm 1,70 \\
47,88 \pm 0,88\end{array}$ & 0,02 \\
\hline
\end{tabular}

Legenda: N: número de alunos;\%: distribuição percentual;e.p.: erro padrão. Fonte: Dados da pesquisa. 
Estudos epidemiológicos têm mostrado que há maiores índices de ansiedade em amostras jovens do que na população mais velha e que a maioria dos indivíduos afetados são do gênero feminino (JANSEN et al., 2011). De acordo com pesquisas americanas, as mulheres possuem maior propensão para desenvolver determinados distúrbios como transtorno obsessivo compulsivo e transtorno de estresse pós-traumático (KESSLER et al., 1994; GOLDSTEIN et al., 2016), o que pode sugerir o fato de que o gênero feminino possui um traço de ansiedade mais marcante que o gênero masculino.
0 fato de este estudo ser direcionado a uma população especifica no contexto acadêmico, foi possível avaliar criteriosamente o perfil dos estudantes quanto a área de atuação acadêmica e profissional. Além disso, é importante destacar que nessa pesquisa o quantitativo de sujeitos do gênero feminino foi maior do que o gênero masculino. Uma vez que no curso de Enfermagem historicamente há um predomínio da presença feminina.

Tabela 2 - Análise descritiva das variáveis avaliadas nos questionários aplicados

\begin{tabular}{|c|c|c|c|c|c|}
\hline & Eventos ocorridos & $\mathbf{N}$ & $\%$ & Média \pm e.p. & $\mathbf{p}$ \\
\hline Ansiedade estado & $\begin{array}{l}\text { Não } \\
\text { Sim }\end{array}$ & $\begin{array}{l}90 \\
58\end{array}$ & $\begin{array}{l}61 \% \\
39 \%\end{array}$ & $\begin{array}{l}47,96 \pm 1,13 \\
54,63 \pm 1,24\end{array}$ & 0,001 \\
\hline \multirow[t]{2}{*}{ Ansiedade traço } & $\begin{array}{l}\text { Não } \\
\text { Sim }\end{array}$ & $\begin{array}{l}90 \\
58\end{array}$ & $\begin{array}{l}61 \% \\
39 \%\end{array}$ & $\begin{array}{l}44,63 \pm 1,00 \\
50,25 \pm 1,19\end{array}$ & 0,001 \\
\hline & Já cursou anatomia & $\mathbf{N}$ & $\%$ & Média \pm e.p. & $\mathbf{p}$ \\
\hline Ansiedade estado & $\begin{array}{l}\text { Não } \\
\text { Sim }\end{array}$ & $\begin{array}{c}116 \\
32\end{array}$ & $\begin{array}{l}78 \% \\
22 \%\end{array}$ & $\begin{array}{l}51,18 \pm 0,98 \\
48,37 \pm 1,97\end{array}$ & 0,19 \\
\hline \multirow[t]{2}{*}{ Ansiedade traço } & $\begin{array}{l}\text { Não } \\
\text { Sim }\end{array}$ & $\begin{array}{c}116 \\
32\end{array}$ & $\begin{array}{l}78 \% \\
22 \%\end{array}$ & $\begin{array}{l}47,25 \pm 0,90 \\
45,31 \pm 1,71\end{array}$ & 0,31 \\
\hline & $\begin{array}{l}\text { Já é formado em } \\
\text { curso técnico }\end{array}$ & $\mathrm{N}$ & $\%$ & Média \pm e.p & $\mathrm{p}$ \\
\hline Ansiedade estado & $\begin{array}{l}\text { Não } \\
\text { Sim }\end{array}$ & $\begin{array}{c}119 \\
29\end{array}$ & $\begin{array}{l}80 \% \\
20 \%\end{array}$ & $\begin{array}{l}50,88 \pm 0,94 \\
49,34 \pm 2,28\end{array}$ & 0,49 \\
\hline Ansiedade traço & $\begin{array}{l}\text { Não } \\
\text { Sim }\end{array}$ & $\begin{array}{c}119 \\
29\end{array}$ & $\begin{array}{l}80 \% \\
20 \%\end{array}$ & $\begin{array}{l}47,18 \pm 0,88 \\
45,41 \pm 1,90\end{array}$ & 0,38 \\
\hline
\end{tabular}

Legenda: N: número de alunos; \%: distribuição percentual;e.p.: erro padrão. Fonte: Dados da pesquisa. 
Estudos realizados na Turquia (BATI et al., 2013) mensuraram a ansiedade traço e a ansiedade estado em alunos de medicina, farmácia e odontologia no primeiro dia da aula prática de dissecação. Os resultados da pesquisa apontaram altos escores de ansiedade estado e ansiedade traço, em que a ansiedade estado foi maior nos homens e a ansiedade traço foi mais significativa nas mulheres, assim como o estudo realizado por Gama e outros autores (2008), no qual a ansiedade traço também foi mais significativa nas mulheres.

Apesar dos estudos realizados por Bati e outros autores (2013) e Gama e colaboradores (2008) referirem que há diferença no nível de ansiedade traço e estado em relação aos gêneros masculino e feminino, a pesquisa em questão mostra que não houve alteração da ansiedade quando comparada aos gêneros. Entendemos como uma possível hipótese sobre esse resultado o fato da amostra masculina ter sido em menor quantidade.

Nos estudos de Bati e outros autores (2013), e Ferreira e outros autores (2009), confirma-se a significância do resultado da ansiedade-traço, sendo o primeiro estudo citado com ênfase no alto índice da ansiedade-traço em mulheres, e o segundo estudo citado, independente de variáveis de sexo, corroborando com os resultados desta pesquisa. Além disso, a presente pesquisa apresentou resultados referentes à formação em curso técnico na área da saúde, no qual as médias de ansiedade traço $(p=0,38)$ e ansiedadeestado $(p=0,49)$ relacionadas a esse fator não foram estatisticamente significativas.

Na variável referente ao fato de ter cursado a disciplina anteriormente ou não, também não houve alteração estatisticamente significativa na ansiedade estado $(p=0,19)$ nem na ansiedade traço $(p=0,31)$, ou seja, ter cursado a disciplina de anatomia humana e também atuar profissionalmente como técnico na área da saúde, não influenciou nos níveis de ansiedade dos alunos que se submeteram ao estudo. No entanto, a variável que se tratava dos eventos previamente ocorridos foi estatisticamente significativa tanto na ansiedade estado $(p=0,001)$, quanto na an- siedade traço $(p=0,001)$, o que levaria à hipótese de que a ansiedade estado teria uma relevância estatística, porém a análise indicou um $p=0,14 \mathrm{da}$ ansiedade estado, ou seja, níveis sem alteração. Na literatura pesquisada, não foi encontrado resultados que relacionassem a ansiedade a eventos previamente ocorridos assim como a presente pesquisa.

Um estudo realizado por Carvalho e colaboradores (2015), objetivou investigar os níveis de ansiedade em universitários ingressantes e concluintes de uma Instituição de Ensino Superior do Noroeste do Paraná, no qual se avaliou a ansiedade, dividindo os participantes em grupos de acordo com as áreas de atuação. Em seus resultados, foram vistos que os universitários concluintes dos cursos dos centros de ciências biológicas e da saúde, assim como de exatas, agrárias e tecnológicas apresentaram maiores índices de ansiedade quando comparados aos ingressantes e que a ansiedade estado foi mais significativa nos alunos de forma geral independente do gênero, pois esta variável não foi avaliada, não corroborando com o presente estudo, devido ao fato de que a ansiedade traço foi mais significativa.

Quando equiparado a outros estudos, é possível observar que os níveis de ansiedade-traço em acadêmicos do curso de Enfermagem de uma universidade de Aracaju, é relativamente menor quando comparados aos níveis obtidos em estudos como o realizado em universitários da mesma cidade (GAMA et al., 2008), o que pode levantar a hipótese de que a área de graduação pode influenciar diretamente no estado ansioso dos estudantes. Uma maneira de aferir isso seria avaliar os níveis de ansiedade-traço e estado em acadêmicos de outros cursos e áreas de atuação.

\section{CONCLUSÃO}

De acordo com a metodologia utilizada, pode-se concluir que a disciplina de anatomia humana não gerou ansiedade estado significativa nos discentes do curso de enfermagem. Porém houve uma ansiedade traço significativa presente nos alunos independente de ser 
do gênero feminino ou masculino. Assim como não houve interferência na ansiedade das variáveis analisadas, como o fato de ter cursado a disciplina anteriormente ou ser formado em curso técnico na área da saúde.

\section{REFERÊNCIAS}

BATI, A.H. et al. Anxiety of first cadaver demonstration in medical, dentistry and pharmacy faculty students. Surgical and Radiologic Anatomy, v.35, n.5, p.419-426, 2013.

CARMO, L.S. et al. Stress into Univesity Students Impacts the Learnig Quality. Journal Psychology \& Psychotherapy, v.6, n.2, p.1-4, 2016.

CARVALHO, E. A. et al. Índice de ansiedade em universitários ingressantes e concluintes de uma instituição de ensino superior. Cienc. Cuid. Saude, v.14, n.3, p.1290-1298, 2015.

CORDEIRO, R.A.; FREIRE, V.; Estado-traço de ansiedade e vivências acadêmicas em estudantes do $1^{0}$ ano do Instituto Politécnico de Porto Alegre. Millenium Journal of Education, Technologies, and Health, v.14, n.36, p.1-7, 2016.

FERREIRA, C.L. et al. Universidade, contexto ansiogênicos? Avaliação de traço e estado de ansiedade em estudantes do ciclo básico. Ciência \& Saúde Coletiva, v.14, n.3, p.973-981, 2009.
GAMA, M.M.A. et al. Ansiedade-traço em estudantes universitários de Aracaju (SE). Rev Psiquiatr., RS, v.30, n.1, p.19-24, 2008.

GOLDSTEIN, R.B. et al. The epidemiology of DSM-5 posttraumatic stress disorder in the United States: results from the National Epidemiologic Survey on Alcohol and Related Conditions-III. Soc Psychiatry Psychiatr Epidemio, v.51, n.8, p.1137-1148, 2016.

HIRSH, C.D. et al. Coping strategies of nursing students for dealing with university stress. Revista Brasileira de Enfermagem, v.68, n.5, p.501-508, 2015.

JANSEN, K. et al. Transtornos mentais comuns e qualidade de vida em jovens: uma amostra populacional de Pelotas, Rio Grande do Sul, Brasil. Cad Saúde Pública, v.23, n.3, p.440-8, 2011.

KESSLER, R.C. et al. Lifetime and 12-month prevalence of DSM-III-R psychiatric disorders in the United States: Results from the National comorbidity survey. Arch Gen Psychiatry, v.51, n.1, p.8-19, 1994.

LEITE, C.D. et al. Representações da ansiedade e medo de atletas universitários. Revista Brasileira de Psicologia do Esporte, v.6, n.1, p.1-11, 2016.

SPIELBERGER, C.D.; BIAGGIO, A; NATALICIO, L.F. Manual do Idate. Rio de Janeiro: CEPA, 1979.
Recebidoem: 20 de Janeiro de 2017 Avaliadoem: 30 de Julho de 2017 Aceitoem: 26 de Setembro de 2017

\author{
1 Acadêmica em Enfermagem da Universidade Tiradentes - UNIT/Aracaju- \\ -SE. E-mail: fernanda.mirellyfreitas@gmail.com \\ 2 Acadêmico em Enfermagem da Universidade Tiradentes - UNIT/Aracaju- \\ -SE. E-mail: victor-levi@hotmail.com \\ 3 Doutora Departamento de Psicologia da Universidade Tiradentes - UNIT/ \\ Aracaju-SE. E-mail: meloliviab@gmail.com \\ 4 Doutor Departamento de Psicologia da Universidade Federal de Sergipe - \\ UNIT/Aracaju-SE. E-mail: andre.faro.ufs@gmail.com \\ 5 Doutor Professor pesquisador da pós-graduação de Psicologia e Matemá- \\ tica da Universidade de Aveiro, Portugal. E-mail: hugodealmeida@ua.pt \\ 6 Doutor Instituto de Tecnologia e Pesquisa da Universidade Tiradentes - \\ UNIT/Aracaju-SE. E-mail:, pauloautranlima@gmail.com
}


